\title{
PRODUCTION OF FOCK STATES IN NONLINEAR OSCILLATOR DUE TO PULSED EXCITATION
}

\author{
T. V. GEVORGYAN \\ Institute for Physical Research National Academy of Sciences \\ Ashtarak 2, 0203, Armenia \\ t_gevorgyan@ysu.am \\ A. R. SHAHINYAN \\ Yerevan State University, Alex Manoogian Street 1 \\ Yerevan 0025, Armenia \\ anna_shahinyan@ysu.am \\ G. YU. KRYUCHKYAN \\ Institute for Physical Research National Academy of Sciences \\ Ashtarak 2, 0203, Armenia \\ Yerevan State University, Alex Manoogian Street 1 \\ Yerevan 0025, Armenia \\ kryuchkyan@ysu.am
}

\begin{abstract}
The Fock states are usually generated by using interaction of oscillatory mode with atomic systems driven by a coherent field. We demonstrate that production of Fock states is also realized in the specific operational regimes of a single nonlinear oscillator without any interactions with atomic systems due to its excitation by a series of short Gaussian laser pulses. These results are demonstrated for the case of the strong Kerr nonlinearity and in the presence of dissipation and decoherence.
\end{abstract}

Keywords: Nonlinear oscillator; Fock state; Quantum State Engineering.

\section{Introduction}

Fock states containing a definite number of energy quanta are eigenstates of the oscillatory excitations number operator. They constitute the essence of the quantum nature of light and play a key role in quantum optics. Generation of pure Fock states is a primary task for the applications in areas of quantum-information processing. Particularly, the experimental preparation, manipulation, and detection of photonic Fock states are of great interest for the implementation of quantum communication schemes, involving quantum cryptography, quantum gates, and for fundamental tests of quantum theory Refs. 1-3. Superpositions of Fock states, par- 
ticularly, vacuum and the single-photon state in a certain optical mode can also be used to implement qubits.

The generation of Fock states has been for a long time an important task with some successful attempts Refs. 4-8. Difficulties in the direct generation of oscillatory Fock states relies to the fact that a classical excitation applied to an oscillator or a linear resonator generates a coherent state, nearly indistinguishable from a classical state, but not quantum Fock states. Recently, it has been demonstrated that Fock oscillatory states can be prepared and manipulated by coupling an oscillator with atomic systems. In this way a classical pulse applied to the atomic states creates a quantum state that can subsequently be transferred to the harmonic oscillator. Such a method was used to deterministically generate Fock number states for the mechanical motion of ions in a harmonic ion trap Ref. 4 as well as for creation of photonic Fock states in electrodynamical resonators Refs. 6, 7 and for generation of Fock states in a solid-state system, i.e. superconducting quantum circuit Refs. 9-10.

In addition to these important inputs, in this paper, we demonstrate that production of Fock states can be realized for the specific operational regimes of driven nonlinear oscillator in the presence of dissipation and decoherence, but without any interaction with atomic systems. For this goal we consider nonlinear dissipative oscillator (NDO) periodically excited by a series of short laser pulses with Gaussian amplitude.

It should be mentioned that NDO driven by external forces possess wide range of applications in both fundamental and applied science. In recent years, the development of these devices operated in quantum regime becomes more and more important with the goal to experimentally implement basic quantum optical systems and quantum logic. The efficiency of quantum nonlinear effects requires a high nonlinearity with respect to dissipation. In this direction, the largest nonlinear interaction was proposed in many optical systems. Cavity quantum electrodynamics-based devices have performed strong nonlinear Kerr phase shifts Refs. 1,2. The simple example of NDO provides self-phase modulation dynamics of single mode in lossy cavity involving $\chi^{3}$ medium where anharmonicity comes from photon-photon interaction. Observation of optical-fibre Kerr nonlinearity at the single-photon level has been demonstrated Ref. 3. The largest nonlinear interaction was proposed in terms of electromagnetically induced transparency Ref. 11. Recently, nano-electromechanical systems and nano-opto-mechanical systems based on various nanomechanical oscillators (or resonators) Refs. 12-16 were shown to be capable of realizing giant Kerr nonlinearity. These systems are attracting interest in a broad variety of research areas and for many possible applications. Remarkable giant Kerr nonlinearities were also found in quantum nanosystems Ref. 17 and quantum circuit systems Ref. 18. Superconducting devices based on the nonlinearity of the Josephson junction (JJ) are the other candidates that offer an unprecedented high level of nonlinearity and low quantum noise. It has been shown that for some specific systems, including Cooper-pair box molecule, the ratio between parameter of nonlinearity and damping can vary from 2 to 45,000 for self-Kerr nonlinearity Ref. 18. Thus, driven os- 
cillators with giant nonlinearities are playing an increasing role in theoretical and experimental quantum physics. In this regard, the NDO with time modulated driving force has been studied in the series of the papers Refs. 19-22 in the context of a quantum stochastic resonance Ref. 19, quantum dissipative chaos Refs. 20, 21 and quantum interference assisted by a bistability Ref. 22 .

It is well assessed that in free decoherence regime an anharmonic oscillator leads to nonclassical states. For NDO over transient regime these effects are decreasing due to interaction with environment. This situation has been strongly demonstrated on the Wigner function that is a joint quasiprobability distribution for the position and the momentum of oscillatory mode and can visualize quantum states as negative values in phase-space. Really, surprisingly simple analytical results for the Wigner functions of NDO under monochromatic excitation in steady state have been analytically obtained Refs. 23, 24 that is positive in all ranges of phase space.

Our main result is that in the pulsed regime of NDO generation of Fock states are realized for interaction time intervals exceeding the characteristic time of decoherence. It is demonstrated in the general form without respect to any concrete system. Thus, we demonstrate that for the pulsed regime the Wigner function of oscillatory mode showing ranges of negative values gradually deviated from the Wigner function corresponding to an NDO driven by monochromatic driving.

\section{Model description and implementations}

In this section we describe the system of interest in applications for its possible realizations in experiments. As driving field we consider sequence of the Gaussian pulses separated by the time intervals. The interaction Hamiltonian of nonlinear oscillator in the rotating wave approximation reads as:

$$
H=\hbar \Delta a^{+} a+\hbar \chi\left(a^{+} a\right)^{2}+\hbar f(t)\left(\Omega a^{+}+\Omega^{*} a\right),
$$

while time dependent driving field amplitude is

$$
f(t)=\sum e^{-\left(t-t_{0}-n \tau\right)^{2} / T 2} .
$$

Here $a^{+}, a$ are oscillatory creation and annihilation operators, $\chi$ is the nonlinearity strength, and $\Delta=\omega_{0}-\omega$ is detuning between frequency of the external field $\omega$ and the frequency $\omega_{0}$ of oscillator.

This model seems experimentally feasible and can be realized in several physical systems. In fact, the effective Hamiltonian describes nanomechanical oscillator with $a^{+}$and $a$ raising and lowering operators related to the position and momentum operators of a mode quantum motion,

$$
x=\sqrt{\frac{\hbar}{2 m \omega_{0}}}\left(a+a^{+}\right), p=-i \sqrt{2 \hbar m \omega_{0}}\left(a-a^{+}\right),
$$


where $m$ is the effective mass of the nanomechanical resonator, $\omega_{0}$ is the linear resonator frequency and $\chi$ proportional to the Duffing nonlinearity. One of the variants of nano-oscillators is based on a double-clamped platinum beam Ref. 25 for which the nonlinearity parameter equals to $\chi=\hbar / 4 \sqrt{3} Q m a_{c}^{2}$, where $a_{c}$ is the critical amplitude at which the resonance amplitude has an infinite slope as a function of the driving frequency, $Q$ is the mechanical quality factor of the resonator. In this case, the giant nonlinearity $\chi \cong 3.4 \cdot 10^{-4} s^{-1}$ was realized. Note, that details of this resonator, including expression for the parameter $a_{c}$, are presented in Ref. 26. It is known that single light mode propagated in Kerr media with dielectric constant $\varepsilon$ and $\chi^{(3)}$ susceptibility is described by the effective Hamiltonian (1). In this case the parameter $\chi$ reads as $\chi=\frac{9}{8} \frac{\hbar \omega^{2} \chi^{(3)}}{\varepsilon^{2} S d}$, where $S$ is the cross section area of the beam and $d$ is the envelope width $(27,28)$. The same Hamiltonian describes currentbiased Josephson junction in rotating wave approximation. In this case creation and annihilation operators are expressed in the following terms:

$$
\begin{aligned}
& a=\frac{1}{\sqrt{2}}\left\{\left(\frac{E_{J}}{E_{C}}\right)^{1 / 4} \phi+i\left(\frac{E_{C}}{E_{J}}\right)^{1 / 4} n\right\}, \\
& a^{+}=\frac{1}{\sqrt{2}}\left\{\left(\frac{E_{J}}{E_{C}}\right)^{1 / 4} \phi-i\left(\frac{E_{C}}{E_{J}}\right)^{1 / 4} n\right\},
\end{aligned}
$$

where $n$ is the charging energy, $\phi$ is the phase difference between superconducting elements in Josephson junction, $E_{J}$ is junction energy, and $E_{C}$ is the Coloumb energy. The parameter of nonlinearity and driving amplitude for this case reads as $\chi=-\frac{E_{C}}{48}, \Omega=-\frac{I \Phi_{0}}{8 \sqrt{2} \pi \eta^{3 / 2}}$, where, $I$ is the biasing current, $\Phi_{0}=h / 2 e$ is the superconductiong flux quantum, $\eta=\sqrt{E_{J} / E_{C}}$. The typical values for Josephson junction used in experiments cooled to $20 \mathrm{mK}$ are $\sim 10 \mathrm{GHz}$ for $|0\rangle \rightarrow|1\rangle$ transition frequency and $\sim 10 \mu \mathrm{A}$ for biasing currents, Refs. 29, 18. The possible decay path $\gamma / 2 \pi$ is order of $2.6 \mathrm{MHz}$ and hence $\chi / \gamma$ is 6.9 , Ref. 18 . However, a large non-linearity in the Josephson inductance is obtained by biasing the junction at a current $I$ very close to the critical current.

We consider the dissipation and decoherence on the base of master equation for density matrix of oscillatory mode via the Lindblad operators:

$$
\rho=-\frac{i}{\hbar}[H, \rho]+L_{1} \rho L_{1}^{+}-\frac{1}{2} L_{1}^{+} L_{1} \rho-\frac{1}{2} \rho L_{1}^{+} L_{1},
$$

where $L_{1}=\sqrt{\gamma} a$ are the Lindblad operators, $\gamma$ is a dissipation rate.

\section{NDO driven by monochromatic driving force}

We start discussion of the main idea by considering at first stage NDO under monochromatic excitation. In the absence any driving, the quantized vibration states of nonlinear oscillator are the Fock states which are spaced in energy 
$E_{n}=E_{0}+\hbar \omega_{0} n+\hbar \chi n^{2}$ with $n=0,1, \ldots$. The levels form a anharmonic ladder with anharmonicity that is given by $E_{12}-E_{01}=2 \hbar \chi$. In the case of monochromatic excitation the vibration states can be driven leading to the Rabi oscillations which are accelerated when frequency of driving is detuned from resonance. The exact analytical results for a driven nonlinear oscillator with dissipation included have been obtained in terms of the Fokker-Planck equation in the complex P-representation Refs. 23, 24. In this way, the solution for the Wigner function of oscillatory mode involving quantum noise in all order of perturbation theory and in steady-state regime have been derived

$$
W(\alpha)=N \exp ^{-2|\alpha|^{2}}\left|\frac{J_{\lambda-1}(\sqrt{-8 \alpha \varepsilon})}{\left(\alpha^{*}\right)^{(\lambda-1) / 2}}\right|^{2} .
$$

Here $J_{\lambda}$ is the Bessel function, $\lambda=(\gamma+i \Delta) / \chi, \varepsilon=E / \chi$, and amplitude $\alpha$ is complex c-number variable corresponding to the operator $a$. It is evident that under monochromatic force the NDO cannot generate nonclassical states as the corresponding Wigner function is positive in all phase space. We analyze this result also on the base of numerical simulation of master equation by using quantum state diffusion method Refs. 30, 31. The results for the Wigner function and the populations of $|0\rangle$ and $|1\rangle$ states for the low excitation regime when only the transition between number states $|0\rangle \rightarrow|1\rangle$ are effectively realized are shown in Fig. 1. As we see, the Wigner function displays some modification of Gaussian that corresponds to the statistical mixing of two oscillatory states. The populations of states shows Rabi oscillations for short time intervals that decays in steady-state regime $t>>\gamma^{-1}$. These results are cardinally changed in the pulsed regime.
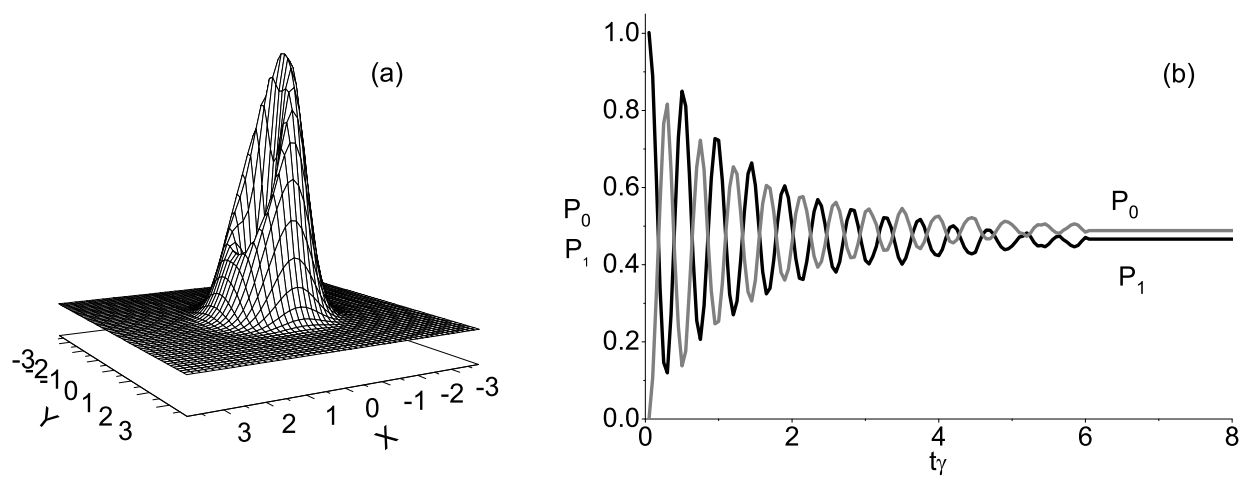

Fig. 1. The Wigner function of oscillatory mode in over-transient regime (a) and the Rabi oscillations of states $|0\rangle$ and $|1\rangle$ (b) for the parameters: $\Delta / \gamma=-15, \chi / \gamma=15 . \Omega / \gamma=6$. 


\section{Production of Fock states}

Below we consider NDO periodically kicked by a series of short laser pulses. We performs numerical calculations based on the method of ensemble averaging of quantum trajectories (see Ref. 30) for arbitrary time-intervals that cover also time intervals exceeding the characteristic dissipation time, $t \gg \gamma^{-1}$. We concentrate on analyzes of quantum regimes for the parameters when oscillatory energy levels are well resolved by consideration of near to resonant transitions between number states $|0\rangle \rightarrow|1\rangle$. Considering the transient regime, $t \ll \gamma^{-1}$, we assume that oscillatory states are initially in the ground state. For the cases of lowest excitation amplitude $\Omega / \gamma$ and when the detuning between driving field main frequency and the frequency of the oscillatory transition, $\delta=\Delta+\chi$ meets the near to resonant condition, effectively only two states $|0\rangle$ and $|1\rangle$ are involved in the Rabi-like oscillations.

We demonstrate below that for above mentioned cases the Fock state $|1\rangle$ can be effectively produced if the time intervals between pulses exceed the duration of pulses. The typical values of time intervals are the following $T / \tau \approx 10^{-1}$. This
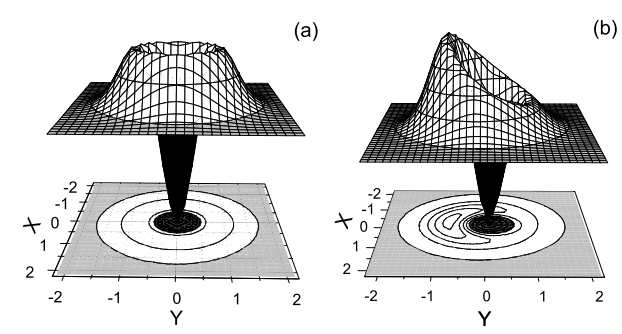

(b)

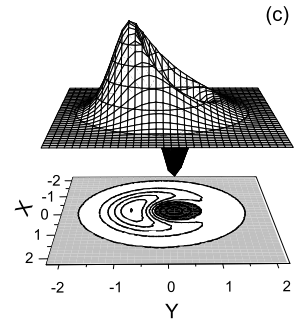

(c)

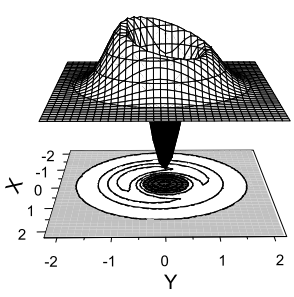

Fig. 2. The Wigner function for pure $|1\rangle$ state (a). The Wigner function evaluation for the parameters: $\Delta / \gamma=-15, \chi / \gamma=15, \Omega / \gamma=6, \tau=5.5 \gamma^{-1}, T=0.4 \gamma^{-1}$; (b) $t=3 \tau-0.4 T$, (c) $t=3 \tau-0.2 T$, (d) $t=3 \tau+T$.

(a)
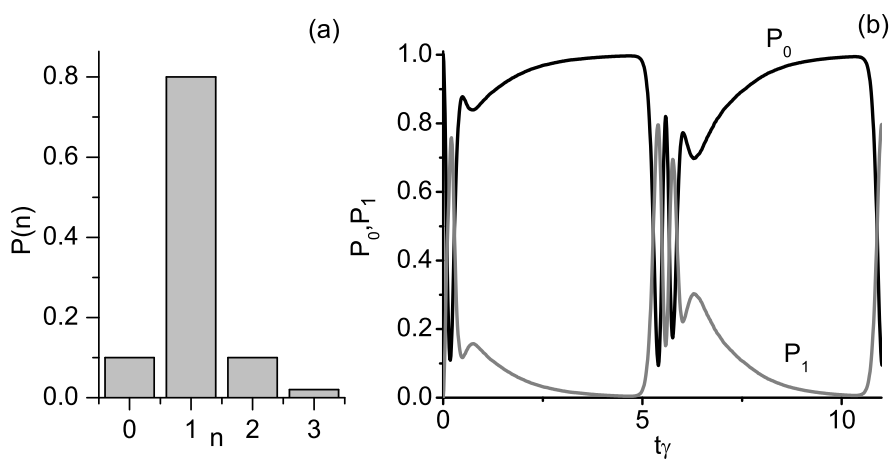

Fig. 3. The excitation number probability distribution (a) and Rabi oscillations (b) for the parameters: $\Delta / \gamma=-15, \chi / \gamma=15, \Omega / \gamma=6, \tau=5.5 \gamma^{-1}, T=0.4 \gamma^{-1}$. The excitation number probability distribution is ploted for $t=3 \tau+T$. 
condition is a request to reach a single excitation level with the population approximately equals to the unity. Under these conditions the non-diagonal elements of the density matrix on the Fock states $|n\rangle$ are negligible in comparison with diagonal elements. The condition of low excitation reads as $|\Omega / \gamma|<|\Delta / \gamma|$. It allows to avoid mixed states, as the external field populates both $|n+1\rangle$ and $|n-1\rangle$ states. Beside this, we note that for effective production of Fock state $\Delta \chi<0$ inequality should be to satisfied. While increasing detuning by its absolute value the probability of state excitation is decreased which means that the external field amplitude should be increased also.

For the pulsed NDO the ensemble-averaged mean oscillatory excitation numbers, the populations of oscillatory states and the Wigner functions are nonstationary and exhibit a periodic time dependent behavior in over transient regime. We plot these quantities for the definite time intervals that exceed the transient time intervals. The typical results for the Wigner functions in the pulsed NDO are plotted in Fig. 2(b-d), for the definite time-intervals within the pulse duration $T$, while the Wigner function of a pure Fock state $|1\rangle$ is depicted in Fig. 2(a). Analyzing the Wigner functions time-evaluation in phase space we conclude the production of Fock state $|1\rangle$ in the pulsed NDO by compared these results with analogous one for the pure $|1\rangle$ state. The Fig. 3(b) shows Rabi oscillations for $|0\rangle$ and $|1\rangle$ states. The maximal weight 0.8 of $P(1)$ (see, Figs. 3(a)) is realized for the time intervals $t=k \tau+T$, $k=0,1,2 \ldots$. In this case the Wigner function of NDO (Fig. 2(d)) is close to pure state Wigner function (See, Fig 2(a)). Indeed, as it was mentioned Fock states have definite number of quanta, which means that in phase space Wigner function should have closed ring signature. Figs. 2(b) and 2(d) show how the rings are becoming closed.

\section{Conclusion}

We have demonstrated the production of oscillatory Fock states in nonlinear oscillator periodically kicked by a series of ultra-short laser pulses in the presence of dissipation and decoherence. Note, that this idea of improving the degree of quantum effects as well as obtaining qualitatively new quantum effects due to appropriately time modulation of open quantum systems, particularly, by trains of pulses was recently has been exploited for formation of high degree continuous variable entanglement in nondegenerate optical parametric oscillator 32 . The effective formation of Fock states has been realized for the definite time intervals within the duration of pulses and has been visualized in phase space by calculation of the Wigner functions of oscillatory mode. This model seems to be experimentally feasible and can be realized in several experimental schemes, including nanomechanical oscillators operated in quantum regime, single light mode propagated in Kerr media with strong nonlinearity and superconducting devices based on the nonlinearity of the Josephson junction. 


\section{References}

1. I. Fushman, et al., Science 320, 769-772 (2000).

2. Q. A. Turchette, C. J. Hood, W. Lange, H. Mabuchi, H. J. Kimble, Phys. Rev. Lett. 75, 4710-4713 (1995).

3. N. Matsuda, R.Shimizu, Y.Mitsumori1, H.Kosaka, and K. Edamatsu, Nature photonics 3, 95 (2009).

4. D. M. Meekhof, C. Monroe, B. E. King, W. M. Itano, D. J. Wineland Phys. Rev. Lett. 76, 17961799 (1996).

5. J. I. Cirac, R. Blatt, A. S. Parkins, P. Zoller, Phys. Rev. Lett. 70, 762765 (1993).

6. B. T. H. Varcoe, S. Brattke, M. Weidinger, H. Walther, Nature 403, 743746 (2000).

7. P. Bertet, Phys. Rev. Lett. 88, 143601 (2002).

8. E. Waks, E. Diamanti, Y. Yamamoto, New Journal of Physics. 8, 4 (2006).

9. M. Hofheinz, et al., Nature 454, 310314 (2008).

10. H. Wang, M. Hofheinz, M. Ansmann, R. C. Bialczak, E. Lucero, M. Neeley, A. D. O'Connell, D. Sank, J. Wenner, A. N. Cleland, and J. M. Martinis, Phys. Rev. Lett. 101, 240401 (2008).

11. A. Imamoglu, H. Schmidt, G. Woods, and M. Deutsch, Phys. Rev. Lett. 79, 1467 (1997)

12. H. G. Craighead. N. Science, 290, 1532 (2000).

13. M. L. Roukes. N. Physics World, 14, 25 (2001).

14. A. N. Cleland. Foundations of Nanomechanics. Springer, Berlin, 2003.

15. K. L. Ekinci and M. L. Roukes, Review of Scientific Instruments 76, 061101 (2005).

16. M. A. Macovei, Phys. Rev. A 82, 063815 (2010).

17. K. Jacobs and A. J. Landahl, Phys. Rev. Lett. 103, 067201 (2009).

18. S. Rebic, J. Twamley, and G. J. Milburn, Phys. Rev. Lett. 103, 150503 (2009).

19. H. H. Adamyan, S. B. Manvelyan and G. Yu. Kryuchkyan, Phys. Rev. A 63, 022102 (2001).

20. G. Yu. Kryuchkyan and S. Manvelyan, Phys. Rev. Letters 88, 094101 (2002); H. H. Adamyan, S. B. Manvelyan and G. Yu. Kryuchkyan, Phys. Rev. E 64, 046219 (2001).

21. G. Yu. Kryuchkyan and S. B. Manvelyan, Phys. Rev. A 68, 0138114 (2003).

22. T. V. Gevorgyan, A.R. Shahinyan, and G.Yu. Kryuchkyan, Phys. Rev. A 79, 053828 (2009).

23. G. Yu. Kryuchkyan, K. V. Kheruntsyan. JEPT 83, 375 (1996); G. Yu. Kryuchkyan, K. G. Petrossian, K. V. Kheruntsyan. JEPT Lett 63, 526 (1996); G. Yu. Kryuchkyan and K. V. Kheruntsyan, Opt. Commun 120, 132 (1996); K. V. Kheruntsyan D. S. Krahmer, G. Yu. Kryuchkyan and K. G. Petrossian, Opt. Commun 139, 157 (1997).

24. K. V. Kheruntsyan, J. Opt. B 1, 225 (1999).

25. I. Kozinsky, H. W. Ch. Postma, O. Kogan, A. Husain and M. L. Roukes, Phys. Rev. Lett. 99, 207201 (2007).

26. H. W. Ch. Postma, I. Kozinsky, A. Hussian, and M. L. Roukes, Appl. Phys. Lett. 86, 223105 (2005).

27. P. D. Drummond and D. F. Walls, J. Phys. A: Math. Gen 13, 725 (1980).

28. L. Mandel and E. Wolf, Optical Coherence and Quantum Optics (Cambridge, UK: Cambridge University Press) chapter 22.

29. R. W. Simmonds, K. M. Lang, D. A. Hite, D. P. Pappas, and John M. Martinis, Phys. Rev. Lett. 93, 077003 (2004).

30. N. Gisin and I. C. Percival, J. Phys. A 25, 5677 (1992); 26, 2233 (1993); 26, 2245 (1993); I. C. Percival, Quantum State Diffusion(Cambridge University Press, Campridge, (2000). 
31. H. H. Adamyan, N. H. Adamyan, N. T. Gevorgyan, T. V. Gevorgyan and G. Yu. Kryuchkyan, Physics of Particles and Nuclei Letters, 5, 161 (2008).

32. H. H. Adamyan and G. Yu. Kryuchkyan, Phys. Rev. A 74, 023810 (2006); N. H. Adamyan, H. H. Adamyan and G. Yu. Kryuchkyan, Phys. Rev. A 77, 023820 (2008). 